\title{
Multi-professional medical record on continuous palliative sedation in an inpatient hospice: a feasibility pilot study
}

\begin{abstract}
Continuous palliative sedation (CPS) is a treatment aimed to relieve refractory symptom in end-of-life patient. The decision to start it involves palliative care unit, patient and caregiver (CG). The objective of this study was to evaluate the feasibility of an instrument to monitor CPS in end-of-life patients. This prospective study was conducted for one year at Bentivoglio Hospice (Bologna, Italy). The multiprofessional medical record (MPMR) is compiled every time a patient begins the CPS. It was composed by four categories of questions on the patient, CG, clinical and pharmacological aspects. MPMR were compiled for 91(16.9\%) patients among the 538 patients who died during the study period. Eighty-nine patients had cancer while two had non-neoplastic disease. The most common refractory symptom was dyspnea $(58.2 \%)$ followed by delirium (36.3\%). Before the onset of the refractory symptom, we openly discussed sedation with 13 patients, while direct patient's agreement to start palliative sedation was possible in 16 cases only. The hospice team evaluated the experience of CPS for CGs. Midazolam was the sedative drug preferred for induction and maintenance of CPS and $64.8 \%$ of patients did not need dosage increases during the maintenance period. A careful evaluation of CPS is important in clinical and pharmacological aspects and in the decision-making process shared by the care team, patient and CG. The MPMR is easy to compile, improves evaluation and follow-up of CPS. It also promotes standard team language and allows a comparison with other settings.
\end{abstract}

Keywords: continuous palliative sedation, hospice, refractory symptom, multiprofessional medical record, midazolam
Volume I Issue 2 - 2017

\author{
Maria Caterina Pallotti,' Maddalena \\ Giovannini, ${ }^{2}$ Cinzia Possenti, ${ }^{2}$ Marco D \\ Alessandro, ${ }^{2}$ Daniela Celin, ${ }^{2}$ Matteo Moroni ${ }^{2}$ \\ 'Department of Experimental Diagnostic and Specialty Medicine, \\ University of Bologna, Italy \\ ${ }^{2}$ Fondazione Hospice MT Chiantore Seràgnoli Onlus, \\ Bentivoglio, Italy
}

\begin{abstract}
Correspondence: Maria Caterina Pallotti, Department of Department of Experimental Diagnostic and Specialty Medicine, School of Medicine, University of Bologna, Via Massarenti 9 , 40138 Bologna, Italy, Tel + 3905 I 2144387, +39347 I59836I, Fax + 39051398973 Email maria.pallotti3@unibo.it, mcaterinapallotti@gmail.com
\end{abstract}

Received: March 24, 2017 | Published: July 03, 2017

\section{Abbreviations: RASS, richmond agitation sedation scale}

\section{Introduction}

Continuous palliative sedation (CPS) is a medical procedure considered a "last resort treatment" to relieve intolerable refractory symptoms in patients facing end of life. ${ }^{1-3}$ CPS is the continuous intentional administration of sedative drugs to decrease patient's levels of consciousness to total unconsciousness. ${ }^{4} 6$ A refractory symptom is a symptom that cannot be adequately controlled by specific symptomatic drugs despite aggressive efforts to identify a tolerable therapy to achieve symptom control without compromising consciousness. ${ }^{7}$ Delirium and dyspnea are the most common refractory symptoms, followed by vomiting, severe pain or convulsions. Sometimes existential distress can be a refractory symptom. Emergency sedation is indicated in the presence of massive bleeding or asphyxiation. ${ }^{2,3,6-10}$ Midazolam is the most frequently administered sedative drug. It belongs to the benzodiazepines, passes the blood brain barrier and diffuses rapidly into the central nervous system with efficacy. It has a short half-life and, consequently, after the first bolus, it must be administered by continuous infusion. Tolerance to midazolam develops frequently requiring an increase of the maintenance dosage of the drug., ${ }^{2,3,6,11-15}$ All other ongoing treatments which effectively control other patient's symptoms are always continued. ${ }^{2}$ The discontinuation of hydration and artificial nutrition are independent of the discussion on CPS..$^{3,10,11,16}$ In addition to its clinical indications, CPS also has ethical implications (such as the decision-making process). CPS does not shorten the patient's life and should not be confused with euthanasia that is instead, the administration of drugs, under explicit request of a patient, at the aim to the intentional ending of his life. ${ }^{6,10,11,17-27}$ During CPS it is important to monitor the sedation, evaluating the patient's clinical condition, consciousness and refractory symptom control in order to establish the need to adjust the drug dosage., ${ }^{2,11,28,29} \mathrm{~A}$ validation scale is recommended for this purpose. The Richmond Agitation Sedation Scale (RASS) is one of the most important scales used in clinical practice. It is well-validated, reliable, easier to use than other scales and less time-consuming, but like other scales the RASS considers only one aspect of sedation: consciousness. ${ }^{11,28}$

The onset of a refractory symptom and the decision to start CPS are delicate issues not only for the patient and family, but also for the Palliative Care Unit. Monitoring all aspects of CPS is essential and widely recommended. ${ }^{2,11,26,30-32}$ Before the start of our study we analyzed the data about the different aspects of the CPS documented in the clinical diary of the patients who died in Bentivoglio Hospice. The Bentivoglio Hospice belongs to the Hospice Seràgnoli Foundation Onlus, an operational structure coordinated by the Isabella Seràgnoli Foundation, Bologna (Italy). This Hospice opened 13 years ago and has thirty bedrooms for patients affected by terminal illnesses. We used the true random number generation system for pulling out in a period of four months (January 2013- April 2013), 80 clinical diary of patients on amount of 220 patients died in Bentivoglio Hospice. Twenty-three patients of 80 patients analyzed, received CPS $(28.7 \%$ 
of the case). We noticed that there is a lack of data in the clinical diary about CPS. For example the steps for the diagnosis of the refractory symptom weren't recorded, the specific treatments administered to control the symptom before the beginning of the CPS, were not always reported in the clinical diary but always in pharmacology report. The RASS scale and the discussion about CPS between palliative care team and the patient were never reported. At the aim of filling this lack we proposed and verified a tool that permitted to report the decision making process of the CPS, simple to use.

\section{Methods}

This is a feasibility pilot study conducted for 12 months at Bentivoglio Hospice (Bologna, Italy). During the study period, every patient admitted to our Hospice was evaluated for enrollment. Inclusion criteria were as follows: a) age $\geq 18$ years, b) inpatient hospice, c) affected by neoplastic or not neoplastic disease, d) onset of a refractory symptom. Exclusion criteria were as follows: a) age $<18$ years, b) a challenging symptom. We develop a multi-professional medical record (MPMR) focusing on the multiple aspects of the CPS: the patients, the caregivers' experience, and the pharmacological aspects of the sedation process as tool for the assessment and follow up of CPS. The MPMR is an ad hoc clinical chart within medical record for monitoring the different aspects of CPS in end-of-life patients with neoplastic or other diseases admitted to the Hospice, who showed refractory symptoms (Appendix 1).

The multi-professional team completed the MPMR whenever a patient started CPS, throughout sedation until the death of the patient. The primary outcome of this prospective study was to evaluate the feasibility to use a multidisciplinary chart for the assessment and follow up of the CPS. The secondary outcomes were to describe the multi-professional dimension of refractory symptom diagnosis, and its decision-making process, to highlight the responsibilitysharing process with $\mathrm{CG}$, and also to promote decision sharing and a homogenous good clinical practice among physicians and nurses belonging to a hospice team. The questions in the MPMR focused on four main aspects of CPS. The first is the patient's diagnosis and the first contact with a palliative care team, the type and the onset of the refractory symptom, and the therapy previously administered to try to control the symptom.

The second aspect is the patient's awareness of his diagnosis and prognosis, including discussions about CPS and the patient's opinion during Hospice hospitalization. In addition, this part of the survey addressed how the patient experienced the onset of the refractory symptom and if he would agree to CPS. The third aspect focused on the caregiver $(\mathrm{CG})$, his perception of the patient's refractory symptom, if he asked to start CPS before the team evaluation, his agreement, any questions about CPS and discussions with the care unit. It is important to specify that we always talked about CPS with the families before patients were admitted to the Hospice. The fourth aspect concerned pharmacologic aspects recording the drug administered as sedative for the induction and maintenance of CPS, drug dosage at the start of the CPS and throughout the procedure until the patient's death. We also specified day 1 (together with the RASS scale of the patient at the start of CPS) and the date of death.

\section{Statistical analysis}

Descriptive analysis was carried out calculating the media age of the study population of the study and the frequency index of all parameters into the MPMR. Univariate analysis was done to the correlation between the dose of sedative drug and age of the patient. We analyzed also in the details the features of the patients and of CGs with which openly discussed of sedation during the recovery, before refractory symptom arose. The time from the beginning of the CPS until the death of the patient was recorded. All data of the MPMR of CPS were collected and analyzed using Microsoft Office-Excel 2010.

\section{Ethical consideration}

The study was approved by the Ethics Committee of Hospice Seràgnoli Foundation Onlus and by the Ethics Committee of ASL Bologna-Imola (Reference number. 14022).

\section{Results}

The study was conducted over one year at Bentivoglio Hospice, from May 2013 to May 2014. In this time frame CPS was administered to 91 out of 583 patients who died at the Hospice (16.9\%). Therefore 91 patients were enrolled in this study and the palliative care team completed 91 CPS multi-professional medical records about the palliative care team completed the MPMR for each one of the patients enrolled. The median age of the study population was 70 years; $67 \%$ were male and $33 \%$ female. Only two patients had non-neoplastic disease (multiple sclerosis, chronic obstructive pulmonary disease), 89 patients had cancer. Lung tumors (37.4\%) and gastrointestinal cancer $(27.4 \%)$ were the most common types of cancer. Patient diagnoses are listed in (Table 1). Refractory symptoms included dyspnea in $58.2 \%$ of patients (53 patients), delirium in $36.3 \%$ ( 33 patients), severe pain $3.3 \%$ (3 patients), existential distress $1.1 \%$ (1 patient), and vomiting $1.1 \%$ (1 patient). Fifty four percent of patients were fully aware of their diagnosis while $27.5 \%$ were fully aware of the prognosis (Table 2). Multiple aspects of the sedation process were openly discussed (what it is, its indication and how it is performed) with 13 patients (14\%) during Hospice admission before the rise of a refractory symptom. One patient did not agree to CPS at first, but when the refractory symptom arose he changed his mind and agreed. Five of these thirteen patients had an early admission to palliative care (Day Hospital access or previous Hospice admission).

Table I Diseases of the patients

\begin{tabular}{lll}
\hline Number patients & $\%$ & Disease \\
\hline 2 & 2.2 & Not neoplastic disease \\
34 & 37.4 & Lung cancer \\
25 & 27.4 & Gastrointestinal cancer \\
II & 12 & Genitourinary neoplasm \\
8 & 9 & Breast cancer, Bone cancer \\
4 & 4.4 & Oropharyngeal cancer \\
3 & 3.3 & Occult Primary \\
3 & 3.3 & Hematologic malignancies \\
I & 1 & Brain tumor \\
\hline
\end{tabular}

The MPMR of the CPS evaluated 90 CG. One patient was alone. During Hospice admission the palliative care team talked to $81 \mathrm{CG}$ (90\%) about CPS. In nine (10\%) cases there was not enough time to discuss CPS with the CGs: in 7 cases the CPS was necessary for the patient in the first week (from day 1 to day 5), while in two case the refractory symptom arose suddenly after a long stay at the Hospice. The frequency of the refractory symptom was reported as always or 
intolerable in $53 \%$ patients, frequently in $42 \%$ and seldom in $5 \%$.At the beginning of CPS only 16 patients $(17.5 \%)$ were in a good enough clinical condition to give their agreement. Only one patient preferred to wait some time before starting CPS.

Table 2 Awareness of the diagnosis and the prognosis of the patients

\begin{tabular}{lll}
\hline & $\begin{array}{l}\text { Awareness of the } \\
\text { diagnosis (Number } \\
\text { patient/\%) }\end{array}$ & $\begin{array}{l}\text { Awareness of the } \\
\text { prognosis (Number } \\
\text { patient/\%) a(num) }\end{array}$ \\
\hline Full & $49(54 \%)$ & $25(27.5 \%)$ \\
Bivalent & $3(3.3 \%)$ & $\mathrm{I} 5(16.5 \%)$ \\
Partial & $\mathrm{I} 2(13.2 \%)$ & $\mathrm{II}(12 \%)$ \\
Absent & $\mathrm{II}(12 \%)$ & $\mathrm{I} 5(16.5 \%)$ \\
Uncertain & $\mathrm{I}(1 \%)$ & $8(8.8 \%)$ \\
Not estimable & $\mathrm{I} 5(16.5 \%)$ & $\mathrm{I} 7(18.7 \%)$
\end{tabular}

When the refractory symptom arose, the palliative care team observed and reported in the MPMR the CG's reaction. $48.9 \%$ of the caregivers showed distress about refractory symptom management: they asked one of the team into the patient's room always $(3 \%)$ or frequently $(47 \%)$. In the $37 \%$ of the case, CG suggested or asked the beginning of the CPS for his patient at the hospice team, before it was recommended. In $98 \%$ of cases, CGs agreed to the start of CPS. One CG appeared uncertain, while in one case the refractory symptom arose during the night and the CG could not be reached by telephone. Midazolam was the sedative drug preferred both for CPS induction (administered in $93.4 \%$ of cases) and maintenance (96.7\%). Haloperidol, diazepam or clothiapine were the other possible sedative drugs administered.

The dose of midazolam was not adjusted throughout CPS in 59 patients $(64.8 \%)$, whereas the dose was increased by $5-20 \mathrm{mg}$ in 19 patients $(20.8 \%)$, by $25-55 \mathrm{mg}$ in seven patients $(7.7 \%)$, and by $70-90$ $\mathrm{mg}$ in two patients. Only one person required the midazolam dose to be increased to $135 \mathrm{mg}$. No dose-related data were available in one patient. We analyzed the correlation between age of the patient and increase of the dose of the sedative drug and we noticed that the patients 51-70 years old required the largest increase of the dose of sedative drug. At the beginning of CPS, the RASS scale was negative in 22 patients $(24 \%)$ and positive in 54 patients $(60 \%)$, with no data available in 14 patients (15\%). The overall survival of patients submitted to CPS was between one hour and 72 hours in $89 \%$ of cases, and more than 72 hours in $10 \%$ of patients (Table 3 ).

Table 3 Duration of sedation

\begin{tabular}{ll}
\hline Hours of CPS & $\mathbf{n}^{\circ}$ Patients $(\%)$ \\
\hline$<$ I hour & $5(5.5)$ \\
I-12 hours & $47(51.6)$ \\
13-24 hours & $9(9.9)$ \\
$25-48$ hours & $16(17.6)$ \\
$49-72$ hours & $4(4.4)$ \\
$73-96$ hours & $4(4.4)$ \\
$97-150$ hours & $4(4.4)$ \\
150-200 hours & 0 \\
$>201$ hours & $2(2.2)$ \\
\hline
\end{tabular}

\section{Discussion}

During a frame time of 12 months of this study in Bentivoglio Hospice 91 patients (of 538 patients who died at the hospice during the same period) underwent CPS. The palliative care team compiled 91 MPMR of the CPS (16.9\%). Eighty-nine patients had cancer while two had non-neoplastic disease. The most common refractory symptom was dyspnea $(58.2 \%)$ followed by delirium $(36.3 \%)$, as reported in literature. ${ }^{6}$ Midazolam was the sedative drug prevalent used for induction and maintenance and the same dosage was maintained throughout all sedation in $64.8 \%$ of patients. This result also confirms that midazolam was the sedative drug preferred for CPS as it is widely demonstrated. ${ }^{2,3,6}$ During hospice care, sedation was openly discussed with 13 patients before the refractory symptom onset, whereas only 16 patients had a clinical condition that they could give their agreement at the start of CPS.

The hospice team evaluated also the experience of the CPS for ninety CGs and the result was that CG worried when the refractory symptoms arise. In fact at the palliative care team, in the $48.9 \%$ of the case, CGs appeared unsatisfactory of the medical and no medical treatment. The overall survival of patients submitted to CPS was between one hour and 72 hours in $89 \%$ of cases, and more than 72 hours in $10 \%$ of patients (Table 3 ). These results evidenced how CPS not influences the prognosis of the patient. ${ }^{10}$ Our study showed the feasibility to use a multidisciplinary chart for the assessment and follow up of the CPS.

A "team tool" for the assessment and follow-up of CPS focused on the multi-professional aspects of refractory symptoms, and on the decision-making process. The MPMR of the CPS serves to share good medical practices among physicians and nurses belonging to a hospice care team by creating a common team language. This tool improves communication leading to a better evaluation of decision-making steps both within the team and with patients and CGs. A secondary outcome is the improvement in responsibility-sharing processes with CGs, providing a real opportunity to empower them and to relieve their stress. Midazolam is the sedative drug most often administered. This study showed also that major dose increases are not required to relieve of the refractory symptom. Patients aged 51-70 years required the largest increase of the dose of sedative drug.

We found that patients can be involved in CPS decision-making and this is easier when patient and team discuss the matter early in the history of the disease. When refractory symptoms arise CGs are usually concerned and often find the care team's work unsatisfactory, requesting prompt sedation before CPS was really indicated. In our experience, CGs invariably agreed when the palliative care unit advised initiating CPS. Our study had a numerous population and this is a relevant feature. Instead the study was performed only in our inpatients hospice and we noticed that at the beginning of the study we were not able to use RASS scale so this date was lost in the $15 \%$ of the case. The CPS multi-professional medical record proposed in this study proved useful for the care team, patients and caregivers: other studies on this topic should be encouraged. The appropriate management and the follow-up of CPS can lower distress of Palliative Care Team leading to reduce patients' suffering and burden of their families.

\section{Acknowledgements}

We thank the Palliative Care Team of Bentivoglio Hospice for the time spent in this study, and specially Marco Paragona for the English 
Language check. A great thanks to Monica Beccaro of Academy of the Science of Palliative Medicine, for her invaluable advises during the draft of this article.

All authors were involved in the concept, design and interpretation of data. The data were collected by M.C.P., M.G., C.P., M.D.A., M.M. The data were subject to preliminary analysis by M.C.P., D.C., M.M. All authors had full access to all of the data of this study. The first draft of the article was written by M.C.P., with redrafting by D.C. and M.M. All authors have seen and approved the final version. The lead author M.C.P. is the guarantor.

\section{Conflict of interest}

The author declares no conflict of interest.

\section{References}

1. Lo B, Rubenfeld G. Palliative sedation in dying patients: "we turn to it when everything else hasn't worked”. JAMA. 2005;294(14):1810-1816.

2. Cherny NI, Radbruch L. European association for palliative care (EAPC) recommended framework for the use of sedation in palliative care. Palliat Med. 2009;23(7):581-593.

3. Cherny NI. ESMO Clinical Practice Guidelines for the management of refractory symptoms at the end of life and the use of palliative sedation. Ann Oncol. 2014;25(Suppl 3):iii143-152.

4. de Graeff A, Dean M. Palliative sedation therapy in the last weeks of life: a literature review and recommendations for standards. J Palliat Med. 2007;10(1):67-85.

5. Kirk TW, Mahon MM. National hospice and palliative care organization (NHPCO) position statement and commentary on the use of palliative sedation in imminently dying terminally ill patients. J Pain Symptom Manage. 2010;39(5):914-923.

6. Maltoni M, Scarpi E, Rosati M, et al. Palliative sedation in end-of-life care and survival: a systematic review. J Clin Oncol. 2012;30(12):13781383.

7. Cherny NI, Portenoy RK. Sedation in the management of refractory symptoms: guidelines for evaluation and treatment. $J$ Palliat Care. 1994;10(2):31-38.

8. Ventafridda V, Ripamonti C, De Conno F, et al. Symptom prevalence and control during cancer patients' last days of life. J Palliat Care. 1990;6(3):7-11.

9. Bush SH, Bruera E. The assessment and management of delirium in cancer patients. Oncologist. 2009;14(10):1039-1049.

10. Maltoni M, Pittureri C, Scarpi E, et al. Palliative sedation therapy does not hasten death: results from a prospective multicenter study. Ann Oncol. 2009;20(7):1163-1169.

11. Maltoni M, Scarpi E, Nanni O. Palliative sedation in end-of-life care. Curr Opin Oncol. 2013;25(4):360-367.

12. Morita T, Chinone Y, Ikenaga M, et al. Efficacy and safety of palliative sedation therapy: a multicenter, prospective, observational study conducted on specialized palliative care units in Japan. J Pain Symptom Manage. 2005;30(4):320-328.

13. Cheng C, Roemer Becuwe C, Pereira J. When midazolam fails. J Pain Symptom Manage. 2002;23(3):256-265.

14. Kohara H, Ueoka H, Takeyama H, et al. Sedation for terminally ill patients with cancer with uncontrollable physical distress. J Palliat Med. 2005;8(1):20-25.
15. Cowan JD, Palmer TW. Practical guide to palliative sedation. Curr Oncol Rep. 2002;4(3):242-249.

16. Rys S, Mortier F, Deliens L, et al. Continuous sedation until death: moral justifications of physicians and nurses--a content analysis of opinion pieces. Med Health Care Philos. 2013;16(3):533-542.

17. ten Have H, Welie JV. Palliative sedation versus euthanasia: an ethical assessment. J Pain Symptom Manage. 2014;47(1):123-136.

18. Materstvedt LJ, Clark D, Ellershaw J, et al. Euthanasia and physicianassisted suicide: a view from an EAPC Ethics Task Force. Palliat Med. 2003;17(2):97-101.

19. Anquinet L, Raus K, Sterckx S, et al. Similarities and differences between continuous sedation until death and euthanasia-professional caregivers' attitudes and experiences: a focus group study. Palliat Med. 2013;27(6):553-561

20. Sykes N, Thorns A. Sedative use in the last week of life and the implications for end-of-life decision making. Arch Intern Med. $2003 ; 163(3): 341-344$.

21. Morita T. Differences in physician-reported practice in palliative sedation therapy. Support Care Cancer. 2004;12(8):584-592.

22. Chiu TY, Hu WY, Lue BH, et al. Sedation for refractory symptoms of terminal cancer patients in Taiwan. J Pain Symptom Manage. 2001;21(6):467-472.

23. Vitetta L, Kenner D, Sali A. Sedation and analgesia-prescribing patterns in terminally ill patients at the end of life. Am J Hosp Palliat Care. 2005;22(6):465-473.

24. Stone P, Phillips C, Spruyt O, et al. A comparison of the use of sedatives in a hospital support team and in a hospice. Palliat Med. 1997;11(2):140 144.

25. Barathi B, Chandra PS. Palliative Sedation in Advanced Cancer Patients: Does it Shorten Survival Time? - A Systematic Review. Indian J Palliat Care. 2013;19(1):40-47.

26. Bruera E. Palliative sedation: when and how. $J$ Clin Oncol. 2012;30(12):1258-1259.

27. Morita T, Tsunoda J, Inoue S, et al. Effects of high dose opioids and sedatives on survival in terminally ill cancer patients. J Pain Symptom Manage. 2001;21(4):282-289.

28. Arevalo JJ, Brinkkemper T, van der Heide A, et al. Palliative sedation: reliability and validity of sedation scales. J Pain Symptom Manage. 2012;44(5):704-714

29. Brinkkemper T, van Norel AM, Szadek KM, et al. The use of observational scales to monitor symptom control and depth of sedation in patients requiring palliative sedation: a systematic review. Palliat Med. 2013;27(1):54-67.

30. Fainsinger RL. Palliative sedation-still a complex clinical issue. Curr Oncol. 2014;21(2):62-63.

31. Maltoni M, Miccinesi G, Morino P, et al. Prospective observational Italian study on palliative sedation in two hospice settings: differences incasemixes and clinical care. Support Care Cancer.2012;20(11):2829-2836.

32. McKinnon M, Azevedo C, Bush SH, et al. Practice and documentation of palliative sedation: a quality improvement initiative. Curr Oncol. 2014;21(2):100-103. 